\title{
Criminologie
}

\section{Vers l'établissement de normes de qualité de services dans les centres d'accueil du Québec}

\section{Gilles Langelier}

Volume 11, numéro 2, 1978

Normes et politique criminelle

URI : https://id.erudit.org/iderudit/017092ar

DOI : https://doi.org/10.7202/017092ar

Aller au sommaire du numéro

Éditeur(s)

Les Presses de l'Université de Montréal

ISSN

0316-0041 (imprimé)

1492-1367 (numérique)

Découvrir la revue

Citer ce document

Langelier, G. (1978). Vers l'établissement de normes de qualité de services dans les centres d'accueil du Québec. Criminologie, 11(2), 76-87.

https://doi.org/10.7202/017092ar d'utilisation que vous pouvez consulter en ligne.

https://apropos.erudit.org/fr/usagers/politique-dutilisation/ 


\section{VERS L'ETABLISSEMENT DE NORMES DE QUALITÉ DE SERVICES DANS LES CENTRES D'ACCUEIL DU QUÉBEC}

Gilles Langelier

Depuis nombre d'années, la qualité des services dispensés par les centres d'accueil du Québec fait l'objet de discussions, singulièrement quand les centres d'accueil occupent la manchette. On réclame, quelquefois à grands cris, des mécanismes pour évaluer la qualité du travail des centres d'accueil et des normes auxquelles ces établissements devraient correspondre. Cependant, au-delà des vœux pieux et des dissertations stériles, les propositions concrètes et constructives sont rares. Les centres d'accueil n'en sont pas moins soumis à de fortes pressions pour assumer une quantité de services sans avoir les moyens minima pour évaluer la qualité de leur action. Les revendications nombreuses de dirigeants de centres d'accueil publics, auprès de l'État, en faveur d'une préoccupation face à la qualité des services, restent souvent lettres mortes devant les impératifs administratifs du fonctionnement d'un système qui doit éviter de donner prise aux critiques de l'opinion publique.

Mais, et heureusement, la société change elle aussi. Elle ne se contente plus de sortir du rang ses jeunes marginaux ou « anormaux»; elle exige que les organismes auxquels elle les confie s'acquittent avec le plus de qualité possible de leur mandat. Enfin nous pourrons pour un moment cesser de parler de « input » ou de « output », comme s'il s'agissait d'une chaîne de montage, pour commencer à regarder, j'allais dire évaluer, ce qui se passe entre ces deux opérations.

Un mouvement d'élaboration de normes de qualité de services dans les établissements de santé et de services sociaux est véritablement engagé et son ampleur est telle qu'il est maintenant irréversible. D'ailleurs, une étude récente sur le sujet ${ }^{1}$ démontre éloquemment la somme d'énergie qu'on y investit, que ce soit aux États-Unis, en Alberta ou en Ontario. Au Québec, l'Association des centres d'accueil du Québec (A.C.A.Q.), dûment mandatée

1. Maurice Cusson et Danielle Laberge-Altmejd, \&es normes de l'intervention auprès des jeunes mésadaptés », C.I.C.C. Ecole de criminologie, Université de Montréal, 1977. 
par ses établissements-membres, est entrée de plain-pied dans ce mouvement depuis deux ans maintenant. Stimulée en cela par les demandes nombreuses et incessantes des centres d'accueil, de la parution du "Rapport Batshaw ${ }^{2}$ et de l'annonce, en février 1976, par le ministre des Affaires sociales de l'époque de la création d'un Conseil de l'agrément des services pour l'enfance mésadaptée (C.A.S.E.M.) ${ }^{3}$, l'A.C.A.Q. a mis à la tâche un comité d'étude ayant pour mandat principal d'élaborer des normes avec et pour les centres d'accueil de réadaptation. Ce texte veut présenter l'ensemble du cheminement suivi par ce comité d'étude et les principales pistes de réflexion qui se dégagent des travaux faits jusqu'à maintenant.

\section{L'Association des centres d'accueil du Québec}

L'A.C.A.Q. est née le $1^{\text {er }}$ juillet 1974, de la fusion de l'Association des Centres d'accueil pour adultes (A.C.A.A.) et de l'Association provinciale des institutions pour enfants (A.P.I.E.).

Elle poursuit les objectifs suivants :

- Regrouper tous les centres d'accueil à but non lucratif, pour la promotion de leurs intérêts et la défense de leurs droits ;

- Représenter les établissements-membres auprès des divers paliers gouvernementaux, groupements professionnels et syndicaux, en collaboration avec les organismes impliqués ;

- Fournir aux membres des services conseils pour l'élaboration et la mise en application de normes de fonctionnement adéquates ;

- Promouvoir la recherche, la formation et le perfectionnement et constituer une banque de documentation pour les membres.

L'A.C.A.Q. compte 320 établissements-membres répartis sur tout le territoire du Québec. Suivant les spécifications de la loi sur les services de santé et les services sociaux (chap. 48 1971), 215 établissements-membres ont une vocation d'héberge-

2. M.G. Batshaw et collaborateurs, « Rapport du comité d'étude sur la réadaptation des enfants et adolescents placés en centre d'accueil $\gg$, Ministère des Affaires sociales du Québec, 1975.

3. Claude E. Forget, "Position du Ministère des Affaires Sociales suite au rapport du comité d'étude sur la réadaptation des enfants et adolescents placés en centres d'accueil », Montréal, 1976. 
ment (personnes âgées) alors que 105 ont une vocation de réadaptation (secteur enfance). L'ensemble de ce réseau de services dessert environ 30000 bénéficiaires en utilisant les ressources de près de 20000 personnes.

\section{L'étude de l'A.C.A.Q. sur les normes}

Au début de ses travaux, en septembre 1976, le comité d'étude de l'A.C.A.Q. sur les normes a été confronté aux principales questions suivantes :

- En quoi consiste une qualité de services dans le domaine des sciences humaines en général et dans un centre d'accueil de réadaptation en particulier ?

- Qu'y a-t-il de vraiment caractéristique dans un centre d'accueil qui offre une «bonne qualité de services?

- Comment évaluer la qualité des services dispensés par un centre d'accueil de réadaptation ?

- Qu'est-ce qu'une norme de qualité de services?

- Sur quelles dimensions du centre d'accueil doivent prioritairement porter des normes de qualité de services?

Sans avoir la prétention d'avoir toutes les réponses à ces questions d'envergure, notre groupe de travail est en mesure de fournir certains éléments qu'on trouvera dans cet article.

Le comité s'est également donné un certain nombre de principes pour encadrer son action et demeurer fidèle à l'esprit du mandat confié :

- Pour atteindre les résultats escomptés, une norme ne peut être imposée mais souhaitée et acceptée par ceux qui auront à l'appliquer. La norme perd son dynamisme si son acceptation doit se faire aveuglément ; il importe d'abord d'être capable de bien voir le contexte dans lequel elle s'insère et surtout, de se voir dans ce contexte, de la façon la plus réaliste possible.

- Il est primordial que les centres d'accueil aient les moyens et la capacité d'évaluer eux-mêmes leur action et la qualité de cette action, avant de se faire imposer des normes et d'y être confrontés. 
- Les normes devront constituer un ensemble dynamique, évolutif et collé à la pratique. Elles seront donc établies à partir de la réalité existante et à cette fin, les centres d'accueil devront être très impliqués dans leur élaboration.

Une fois posés, ces principes nous amenèrent à élaborer un plan de travail dont les principales étapes consistèrent à :

- déterminer, parmi l'ensemble des dimensions du centre d'accueil, celles qui permettent davantage de cerner la qualité des services qui sont offerts et conséquemment, celles qui seront prioritairement l'objet d'une élaboration des normes de qualité.

- définir la notion des normes et ses diverses facettes.

- préciser les principes et les moyens pour évaluer la qualité des services dispensés dans les centres d'accueil et ce faisant, déterminer la participation des centres d'accueil à l'évaluation des services de même qu'à l'élaboration et l'implantation des normes.

\section{Le centre d'accueil : une réalité complexe}

Le centre d'accueil est un milieu humain dont la mission unique et ultime est de venir en aide à des personnes en difficulté. C'est là le mandat qui lui est confié par la société et duquel il doit rendre compte. Le centre d'accueil doit être sans cesse au fait des besoins changeants de la société et se placer dans un état de recherche presque permanent des moyens les meilleurs pour s'acquitter avec qualité de son mandat humaniste et de son rôle social.

La réalité même du centre d'accueil est cependant la principale source de difficulté à son évaluation. C'est une réalité mouvante, changeante, qui donne peu de prise à l'évaluation scientifique et contrôlée. C'est un milieu formé de nombreuses composantes, significatives chacune en elles-mêmes mais aussi, et peut-être davantage, lorsqu'elles sont en interaction. Le centre d'accueil, soucieux de satisfaire les besoins pour lesquels il existe, doit être en recherche continuelle des meilleurs moyens pour le faire. C'est un milieu qui se doit d'être en état de perpétuelle remise en question. Cette capacité de remise en question est même, jusqu'à un certain point, devenu un critère de «santé * du centre d'accueil. Cette réalité n'est pas sans poser plusieurs problèmes lorsque nous devons envisager l'évaluation de la qualité 
des services. Il s'avère nécessaire que l'évaluation devienne une fonction permanente du centre d'accueil.

Mais quelles dimensions de ce système complexe et mouvant convient-il de regarder et de soumettre à l'évaluation pour arriver à déterminer la qualité des services qui y sont dispensés ? Certains éléments apparaissent d'emblée.

Le point de départ, c'est la raison d'être du centre d'accueil, c'est-à-dire la clientèle qu'il reçoit. Une fois bien identifiés les besoins et caractéristiques des personnes qu'il veut aider, le centre d'accueil doit se donner des objectifs précis, concrets et mesurables. Pour les atteindre, il doit pouvoir compter sur des personnes qu'une formation personnelle et professionnelle habilite à mettre en cuvre les programmes et autres moyens les plus en mesure d'aider les gens en difficulté qu'ils ont à assumer. De plus, ces quelques dimensions du centre d'accueil, clientèle-objectif-personnel-programmes, doivent être, chacune et ensemble, imprégnées d'un esprit, de valeurs fondamentales qui seraient incarnées dans une conception de l'intervention qu'on appelle également une philosophie de traitement, une approche rééducative ou une idéologie maîtresse. Cette conception de l'intervention constitue en fait un principe de cohésion dans un milieu voué à la réadaptation. Elle imprègne toutes les fibres du milieu et chaque élément constituant ce milieu doit s'en inspirer. C'est véritablement l'épine dorsale qui donne vie et alimente chaque ramification d'un milieu de réadaptation.

Une recherche ${ }^{4}$ faite pour le compte du comité d'étude sur la réadaptation des enfants et adolescents placés en centre d'accueil affirme :

$\mathrm{Au}$ début de notre intervention dans les établissements, nous avons promptement constaté qu'aucun modèle administratif pur, fondé sur l'application rigoureuse des principes de planification, d'organisation, de direction et de contrôle ne peut s'opérationnaliser dans un contexte de réadaptation sans être orienté, guidé, articulé et inspiré par une idéologie maîtresse, en l'occurrence une philosophie de traitement prédominante (p. 26).

4. Consultation B.T. Inc. « L'organisation et le personnel des centres d'accueil de transition et de réadaptation du Québec », Annexe 3A, Rapport du Comité d'étude sur la réadaptation des enfants et adolescents placés en centres d'accueil, Ministère des Affaires sociales, Québec, 1975. 
et plus bas :

tous les centres à vocation (de) traitement devraient opérer en fonction d'une approche thérapeutique (p. 26).

S'inspirant notamment de ces affirmations, le comité d'étude de l'A.C.A.Q. a adopté l'hypothèse de travail suivante :

Un centre d'accueil qui offre une bonne qualité de services serait celui dans lequel existe une conception de l'intervention clairement identifiée, et qui se retrouve dans la pratique.

Il en résulte le fait que la conception de l'intervention doit occuper une place privilégiée dans le processus d'évaluation et partant, dans celui de l'établissement de normes de qualité.

Ce n'est cependant pas la seule composante du centre d'accueil qu'il importe de regarder pour évaluer la qualité des services. Après étude, nous adoptons ces autres dimensions :

- la classification et la vocation de l'établissement ;

- les objectifs généraux de l'établissement ;

- les programmes ;

- le point de départ de l'intervention ;

- la continuité de l'intervention ;

- les dossiers des bénéficiaires ;

- l'action du personnel ;

- la participation des bénéficiaires et des parents aux objectifs d'intervention ;

- les aspects administratifs.

Ce sont les dix éléments que le comité d'étude a choisi d'utiliser pour explorer la dimension «qualité de services » à l'intérieur d'un centre d'accueil. Ils constitueront par le fait même les premiers éléments sur lesquels porteront les normes de qualité que nous publierons. D'autres éléments pourront ultérieurement s'ajouter au processus.

\section{Les normes: une définition et quelques caractéristiques}

Selon le contexte auquel il est appliqué, le terme «norme * peut revêtir divers sens. Il convient certes de circonscrire le plus précisément possible celui qu'on lui donne afin d'éviter les ambiguïtés qu'une mauvaise compréhension pourrait entraîner. Le sens qu'on lui prête peut également avoir une influence importante sur l'ensemble du travail de définition et d'implantation de normes. 


\section{Définition}

Les travaux menés sur le sujet nous ont conduits à définir une norme comme étant une règle d'action traduisant une valeur et une ligne directrice de conduite. Les normes, dans le cadre qui nous occupe, sont essentiellement des règles que les centres d'accueil se donnent pour préciser les lignes directrices qui les aideront à guider et évaluer leur action. Elles doivent tenir compte de la réalité, et même partir d'elle, mais également, proposer une excellence à viser. En ce sens, les normes possèdent un caractère évolutif, impliquent une continuité et font le pont entre l'acquis et le désirable.

Cusson et Laberge-Altmejd présentent dans leur ouvrage ${ }^{\circ}$ deux types de normes : « minimales > et "optimales ». Même si nous ne sommes pas en total désaccord avec une telle conception, nous préférons considérer un seul type de norme qui soit dynamique et évolutive dans le sens qu'elle cherchera sans cesse à tenir compte des besoins changeants de la société et de l'évolution des centres d'accueil. L'acquis peut changer, de même que le désirable. A elle seule, cette caractéristique illustre bien les possibilités évolutives que devront avoir les normes élaborées et milite en faveur de l'adoption d'un seul type de norme.

\section{La nécessité d'avoir des normes}

La nécessité pour les centres d'accueil d'avoir des normes de qualité de services est justifiée à partir de deux volets principaux : en regard de la société et en regard du fonctionnement même du centre d'accueil.

En ce qui concerne la société, il convient de remarquer le développement d'un plus grand intérêt du public pour connaître et comprendre la vocation actuelle du centre d'accueil et partant, du travail qui s'y fait. Nous pouvons parler de l'éclosion d'une conscience sociale plus grande chez les profanes, qui coïncide avec le développement d'une ouverture plus concrète du centre d'accueil sur la communauté en tant que ressource avec laquelle il convient de composer. Cet état de fait doit inciter le centre d'accueil à se donner des moyens pour définir, évaluer et contrôler son action. Cette véritable évolution sociale ne fait que

5. \& Les normes de l'intervention auprès des jeunes mésadaptés ", 
confirmer et par là, stimuler ce mouvement discret, continu et maintenant irréversible de l'établissement de normes dans les centres d'accueil.

Les normes sont aussi nécessaires en regard du fonctionnement même du centre d'accueil. Considérées dans leur aspect dynamique, elles peuvent représenter une sorte de modèle qu'un milieu se donne et auquel il peut référer pour évaluer et planifier son action. En cela même, elles peuvent constituer pour les divers intervenants d'un milieu, un point de référence et une source de cohésion. Elles stimulent un milieu à se donner des objectifs de qualité de services et lui indiquent des moyens pour y parvenir. Elles peuvent permettre aux éducateurs de situer la qualité de leur action et par là, de s'améliorer.

\section{Avoir des normes présente certains dangers}

Cusson et Laberge-Altmejd font largement état des risques inhérents à l'établissement de normes de qualité. Les remarques qu'ils font sont pertinentes et nous y souscrivons entièrement.

Même si nous réussissons à élaborer les normes les plus parfaites, un danger demeure : celui de les utiliser comme un modèle rigide, une sorte de gabarit idéal qui devient le moule auquel tout sera assujetti.

On peut également perdre de vue l'esprit de la norme et l'utiliser comme un moyen pour juger sommairement d'une situation et par là, se priver de toute la qualité de la réflexion que cette norme pourrait susciter. L'objectif ultime d'un recueil de normes doit être d'aider les centres d'accueil à acquérir, maintenir et développer une qualité de services, à la hauteur des attentes de la société et des besoins des personnes en difficulté. Pour éviter qu'elles ne viennent paralyser la créativité, empêcher l'innovation et retarder l'évolution nécessaire, les normes ne doivent pas être des simples règlements plus soucieux de faire fonctionner un système et d'en contrôler l'efficacité que de servir les intérêts des milieux complexes que nous décrivions précédemment.

Il s'avère de première importance que les principaux acteurs de l'action soient très impliqués dans l'élaboration, l'implantation et l'utilisation des normes. Dans notre esprit, c'est d'abord pour eux et pour les personnes qu'ils ont à aider qu'elles devront exister. 
L'évaluation de la qualité des services

Tout notre processus de définition, d'élaboration et d'implantation de normes repose essentiellement sur l'évaluation même de la qualité des services. Nous nous devons donc de préciser notre conception de l'évaluation de la qualité des services en centre d'accueil, de ses caractéristiques, du rôle du centre d'accueil dans l'évaluation de ses services et de situer la place de cette conception dans le processus d'élaboration des normes.

Les centres d'accueil, comme toute entreprise, ont besoin d'évaluer la qualité des services qu'ils dispensent. Plusieurs raisons justifient ce besoin. Citons entre autres les suivantes :

- pouvoir identifier et apprécier les résultats obtenus ;

- exercer une planification éclairée ;

- assurer une coordination vigilante entre les divers éléments constituant le milieu de réadaptation ;

- augmenter leurs connaissances dans le secteur de la réadaptation en tirant le meilleur profit possible de leur expérience ;

- accroitre et parfaire leurs moyens d'influence sur les personnes qu'ils ont à aider et, de plus en plus, sur une communauté donnée ;

- se donner un certain recul sur l'action quotidienne engagée ;

- identifier l'écart qui peut exister entre les prévisions et les résultats et avoir des moyens pour expliquer les causes et pour pouvoir ajuster leur action.

\section{Définition de l'évaluation}

Evaluer la qualité des services dans un centre d'accueil ne peut se limiter à mesurer une efficacité ou un rendement. Il faut déborder et englober ce concept de mesure. Il ne s'agit pas simplement de cerner un fait et de l'isoler de son contexte pour mieux l'étudier. L'évaluation doit porter sur le contexte même à l'intérieur duquel se trouve le fait en question et cherchera à cerner la nature de ses interrelations avec un ensemble identifié. En ce sens, l'évaluation se réfère essentiellement à des critères et appelle, par sa comparaison à des normes adoptées, des jugements, des interprétations, des hypothèses et des constatations. 
Les données recueillies par l'évaluation doivent servir à situer l'action mais également à la modifier sans cesse. Il ne s'agit pas que de « prendre une photo pour les archives ».

Le centre d'accueil, de par sa vocation autant sociale que thérapeutique, ne peut se permettre de statu quo. Divers dangers guettent le centre qui « s'arrête » : la stagnation, l'autosuffisance, la complaisance dans un système plus soucieux de s'autoconserver que de servir la société et les jeunes en difficulté.

L'évaluation des services est un moyen pour identifier une qualité donnée, pour mesurer la valeur des moyens employés, pour réajuster l'action exercée et foumir des points de référence aux animateurs du milieu et responsables de son fonctionnement et de son évolution.

\section{À la recherche d'un modele d'évaluation}

L'évaluation de la qualité des services dans un centre d'accueil ne peut se faire avec un test qu'on applique sans tenir compte des nombreux et importants facteurs qui influencent, chacun à leur façon, le fonctionnement du centre d'accueil. À ce niveau, les points de référence sont rares et le comité d'étude de l'A.C.A.Q. a été placé devant la nécessité de créer un modẻle adapté aux objectifs poursuivis par l'étude. Il nous fallait trouver un modèle qui aide le centre d'accueil à évaluer certaines de ses dimensions, la nature des interactions entre ces dimensions et l'importance de ces interactions dans un ensemble.

De plus, nous voulions que cette évaluation favorise un meilleur fonctionnement du centre d'accueil, transforme certaines mentalités, mène une plus grande cohésion, influence le développement de certains services, améliore les services existants et fournisse aux centres d'accueil des paramètres de planification.

Fidèles à nos principes de départ, nous avons opté pour l'approche de l'auto-évaluation. Nous avons donc construit un instrument, soit un long questionnaire qui explore chacune des dix dimensions du centre d'accueil que le comité d'étude a choisi comme étant pertinentes à la qualité des services dispensés. La majorité des questions sont ouvertes et les réponses appellent évidemment beaucoup de subjectivité. Cette ligne d'action a de quoi étonner quand on considère l'importance accordée à la recherche de l'objectivité en sciences humaines. Sans refuser ni 
minimiser sa valeur, nous dirons que l'objectivité ne constitue pas pour nous, du moins pour l'instant, un facteur de premier plan ni même un objectif visé. Ce qui nous importe davantage, c'est de connaître la situation actuelle dans les centres d'accueil, telle que perçue et vécue par les personnes responsables de ces milieux. Il nous importe également de faire vivre à ces personnes une démarche d'auto-évaluation de leurs services. Il ne s'agit plus d'experts qui viennent dire aux «gens dans l'action» ce qui en est de leur réalité. Ce sont ces mêmes "gens dans l'action》 qui apprennent à regarder et lire leur réalité et qui se donnent ensuite les moyens de l'améliorer.

Il s'avère primordial que les centres d'accueil soient responsables de l'évaluation de la qualité de leurs services. Pour saisir dans ce qu'elle a d'essentiel toute la réalité vivante dans son dynamisme et sa complexité, il nous apparaît nécessaire et fondamental de s'en remettre aux acteurs de la situation. Par là, nous affirmons que l'évaluation ne peut être dissociée de l'action.

Ce modèle d'évaluation poursuit un deuxième objectif en regard de la problématique qui nous occupe : celui de recueillir un matériel à partir duquel seront élaborées les normes de qualité de services. Nous avons demandé à tous les centres qui ont reçu l'instrument d'auto-évaluation de nous retourner, sous le couvert de la confidentialité la plus stricte, leur instrument dûment complété. La réponse des centres d'accueil à cette demande est des plus positive. Elle traduit éloquemment l'intérêt des centres d'accueil en regard de l'établissement des normes et leur satisfaction quant à la valeur de la démarche d'évaluation que nous leur avons fait vivre.

Ce matériel fournira au comité d'étude deux sources de normes : celles qui se rattacheront à chacune des dix dimensions choisies et celles qui émergeront des interactions entre ces dimensions.

\section{Conclusion}

Beaucoup serait encore à dire sur le travail fait dans ce dossier, les difficultés que nous avons rencontrées et celles que nous anticipons dans l'analyse de l'imposant matériel que les centres d'accueil nous ont fait parvenir. Qu'il suffise simplement de rappeler que nous refusons un recueil de normes du style * petit caté- 
chisme , fait par des « experts , qui viennent photographier les centres d'accueil avec des appareils de fabrication étrangère! L'élaboration et l'implantation de normes doit procéder d'une évaluation des services, d'une remise en question des centres d'accueil et être le fait d'une collaboration vécue et d'une concertation réelle entre les animateurs des centres d'accueil, le ministère responsable et des spécialistes habilités à utiliser les expériences acquises et à traduire la pensée des personnes qui sont dans l'action. 